\title{
Competence in Medical Practice as Perceived by Malaysian Medical Interns: A Measurement Invariance Analysis
}

\author{
Abideen Adeyemi Adewale*, Noor Hazilah Abd. Manaf, Azura Omar \\ International Islamic University Malaysia \\ *abidewale@iium.edu.my
}

\begin{abstract}
This study investigates the perception of Malaysian housemen on what the indicators of competence are in the medical practice. This derives from the need for the interns to have the requisite skill needed to make them competent in the post-medical school medical practice. A survey questionnaire was administered on all 2,046 housemen in all medical schools across Malaysia as at April 30, 2008 when data was collected. Data so obtained were subjected to statistical analysis based on $\mathrm{N}=1213$ using both the structural equation modeling via SPSS AMOS 18 software. Nine skills were identified in all. These are interpersonal, IT, patient management, basic, holistic, science, ethics, coping, and clinical skills. While all the skills were found to be statistically significant, no factorial invariance was observed when the data is split into demographic divides.
\end{abstract}

Keywords: Internship, competence, factorial invariance, practice

\section{Introduction}

To become a medical practitioner arguably takes more training time, examinations, and practices than any other profession. This is necessitated by the need to produce competent health care practitioners capable of displaying cognitive, technical, and personal skills for meeting the expectations of all stakeholders including the government, hospital management, patients and the society at large (Aggarwal, Grantcharov \& Darzi, 2007). These attributes, expectedly should be present in any medical doctor. Nonetheless, it is the ability to combine these skills and sensitivities of an applied scientist to the reflective capabilities of a medical humanist that distinguishes a good medical doctor from both the poor and the bad ones (Hurwitz \& Vass, 2002). ${ }^{1}$ A good medical doctor should ideally demonstrate the possession of the requisite skills needed to cope with changes in the medical industry. The field of medicine has, over the last fifty years undergone an explosion in medical and technical knowledge transitioning from the 'art' to the 'science' of medicine (McLaughlin \& Kaluzny, 1999). The changing healthcare landscape is not confined to the way hospitals are managed alone. Patients' profile is also changing. The internet and information revolution has empowered patients and they are no longer content with the attitude that 'doctor knows best'. Today's patients come with certain expectations and demands of their own; departing towards a more participative approach in decision-making that concerns their health conditions, rather than simply passive receivers of healthcare services. Moreover, in a multi-ethnic and multi-religious country like Malaysia, such professionalism is expected to reflect respect, sensitivity and responsiveness to culture, age, religion, gender etc. Sivalingam (2004) succinctly stated:

The need to promote humanistic values and to detest dehumanizing processes is vital. Teaching good medical practice, and inculcating the values and behavior expected by society, and sensitivity to religion and culture are needed, especially when students may have been monolithic in their exposure to different cultures in Malaysia because of their early education. Shapiro (2008), therefore, noted that a balance of some sort is needed to mitigate the varying perspective and expectations of professional conducts in medicine within a diverse cultural setting. What may be medically justified and necessary may just be religiously or culturally

\footnotetext{
${ }^{1}$ While the poor doctors are prone to committing unintended medical mistakes arising from their gross incompetence, the bad doctors, though possess commendable and requisite medical competence lack good intentions and have serious defects of moral agency (Hurwitz \& Vass, 2002).
} 
unacceptable. For instance, Primadasa et al., (2007) noted the difference in exposure to clinical procedures in Obstetrics and Gynecology between male and female interns given the value system in the Arabian cultural setting in Kuwait. Given these changes and challenges facing the industry, healthcare professionals produced by medical schools and colleges have to be relevant to meet the expectations of the various healthcare stakeholders. For that reason, it is imperative for Governments and their health agencies to ensure that medical schools and colleges in a country are producing competent professionals who have acquired the requisite skills, knowledge and expertise for medical practice. With the benefit of hindsight, it may be safe to presuppose that the low or unsatisfactory performance of some physicians is a manifestation of their incompetence. A hypothesis, therefore, may be that once the causes of incompetence among the physicians can be identified and remedied, their performance would also be consistent with the expectations of all the healthcare stakeholders. Competence is, therefore, a key issue for determining the preparedness of medical interns for; and subsequent performance during medical practice later in life. The objective of this paper, therefore, is to identify the core competence indicators in medical practice from the point of view of medical interns. The remainder of this paper includes the materials, methods, results and conclusion of the study.

\section{Meaning of Competence}

Competence as a concept has a great diversity behind it. It is an issue that exercises a growing appeal among medical experts and commentators alike especially as it relates to the performance of medical personnel. It enjoys a profile of meanings and conceptualizations as a celebration of the various perspectives from which it can be viewed. In the context of the assessment of the competency of a physician, Epstein (2007) succinctly defines competence in medicine as the habitual and judicious use of communication, knowledge, technical skills, clinical reasoning, emotions, values, and reflection in daily practice for the benefit of the individuals and communities being served. This definition is most apposite for many but basically because of two reasons. First, it is comprehensive and aligns well with the current clamor for a blend of the attributes of an applied scientist and a medical humanist in the modern day 'good doctor'. Second, it reflects the relative importance of the six general competencies that to a reasonable extent frame and define the substance of medical practice independent of specialty and delivery model as championed by the Accreditation Council for Graduate Medical Education (ACGME) (Leach, 2002). Competence in medicine may also be viewed as a latent talent, which can be aroused and further developed in an environment that permits the nurturing of such innate talents. According to Neeraj, Burkhalter and Cooper (2001), it refers some particular knowledge, traits, skills, and abilities that reside in an individual, which helps him/her to exercise self-control and confidence in understanding the content and context of a situation. As such, he can apply his skills and knowledge in a way that reflects in conduct and outcome, the mastery of the procedures involved owing to innate or acquired expertise. As succinctly argued, further by Leach (2002), competence is a habit. It is a manifestation of consistent ability that yields desirable outcomes based on design rather than by happenstance. Corroborating this view, Epstein (2007) stated that "competence is not an achievement but rather a habit of lifelong learning in which assessment plays an integral role in helping physicians identify and respond to their own learning needs."

This perhaps is because as physicians transit along various stages from novice to experts, they are confronted by professional challenges that demand that they exhibit context-based professional behavior rather than appeal to the rule-based approach only. As Leach (2002) would argue, "rules become subliminal and are integrated with intuition." In this case, competence reflects a physician's ability to appeal to his instincts and medical rules; and as such also accept a higher level of accountability and responsibility for his professional judgments. In the case of the housemen who are operating at the stage between the novice and competent physician in the medical training cycle, more involvement in; rather than detachment from some context specific medical situations and patients enhances their prospect of becoming 'good doctors' upon their professional independence (Epstein, 2007). Although the relationship between competence and performance in medicine is somehow vague (Neeraj, Burkhalter, \& Cooper, 2001), universality exists in the extant literature of the dependency of performance on competence. The irreversibility of the relationship between the two derives from the fact that performance rarely may not necessarily be a function of competence (While, 1994). According to Epstein (2007) while competence in medicine refer to what a student or physician can do, performance relates to what the student or physician actually and habitually do when not under observation. Therefore, as a major determinant of professional performance (measuring up to a level of 
professional standards and expectations) consequent to adequacy of preparedness for future independent practice during horsemanship, an assessment of the competence of interns is justified. According to Epstein (2007) "such assessment can be formative (guiding future learning, providing reassurance, promoting reflection, and shaping values) or summative (making an overall judgment about competence, fitness to practice, or qualification for advancement to higher levels of responsibility)."

Indicators of Competence in Medical Practice: The contextual nature of competence in; or preparedness for independent medical practice presupposes that quite a plethora of indicators as its determinants exist. In fact, according to Neeraj, Burkhalter, and Cooper (2001), health workers independent of their specialty require a myriad of and harmonized set of skills, traits, knowledge and ability in order to deliver quality medical care and services. As succinctly stated by Marple (2007), the indicators of competence in medicine derive essentially from a thorough research process to evaluate the qualities necessary for a physician to function effectively. The purpose, argued further by Marple (2007) was to stimulate an introspective assessment of how adequately physicians are prepared to rise up to the challenge of the unprecedented dynamic arena of healthcare service rendering and delivery. Each of the six general competences is considered in turn below.

Patient Care: Patients to healthcare institutions can be likened to customers or clients to a typical organization. Thus, satisfying their often-diverse expectations places a great onus on the health workers. This is particularly to the physicians whose function is the pivot around which other health employees' functions rotate. According to Janiciket al. (2007), housemen are faced with the herculean task of attending to patients who vary in their demographical attributes and medical presentations. When a archetypical challenges exist in a multicultural and multi-religious nation like Malaysia, it is understood that the interns need to demonstrate a blend of adequate diagnostic acumen and competence, as well as a refined sense of humanity. Showing adequate and timely empathy towards a patient's plight is an important attribute of a good doctor (Woolfet al., 2007). The interns, therefore, are expected to be compassionate and provide appropriate and effective treatment to patients (Marple, 2007). This according to Janicik (2007) has been shown to have a positive link to the outcome of treatments in patients. In their qualitative study, Watmough, Taylor and Garden (2006) concluded that medical supervisors were unanimous in their assertion that this is a very important indicator of competence in the interns. The study further revealed that the medical supervisors opined that the (Practice Based Learning)) PBL graduates demonstrated a higher level of patient care compared to their traditional curriculum based counterparts. As noted by Neeraj, Burkhalter and Cooper (2001), patients care as an attribute of competence presupposes the possession of medical knowledge. Having compassion for the patients would be insufficient and indeed counterproductive if not predicated on sufficient medical knowledge (Hurwitz \& Vass, 2002). Therefore, possessing the requisite medical knowledge, which forms the base upon which introspection derives is an indispensable competence indicator in medicine.

Medical Knowledge: The imperative of the housemen possessing the requisite basic medical knowledge have always been an important indicator of their competence even before the changing trend towards the PBL curriculum system of medical education. This perhaps underscore the long years of training and variety of examinations that medical students are subjected to in order to gauge their knowledge depth. According to Alexander (2007), for medical knowledge to give a reliable indication of competence and preparedness for medical practice by medical graduates, its adequacy should be considered in the context of acquisition, analysis, and application. In other words, supervisors often assess the medical knowledge depth of their medical supervisees based on their ability to apply knowledge they had acquired from their graduate programs to identify, diagnose and analyze medical presentations with relative ease. The need for medical knowledge was further reiterated by Marple (2007) who stated that "residents must demonstrate knowledge about established and evolving biomedical, clinical, and cognate (e.g., epidemiological and social-behavioral) sciences and the application of this knowledge to patient care." Perhaps, this offers a plausible reason why supervisors accentuate on their interns' basic medical knowledge as an indicator of competence albeit at varying degrees reflecting specialization requirements. According to Watmough, Taylor, and Garden (2006), knowledge as an indicator of competence has sparked a serious controversy among supervisors on its adequacy or otherwise among interns. While some argued against the factual burden arising from trying to instill too much but irrelevant knowledge into the medical curriculum, the dissents hold that such are still 
necessary for the interns to have a broad view of issues, which then can be introspectively applied. Notwithstanding the controversy surrounding this indicator of competence, an apparent unanimity indicate that the supervisors thought the graduates from the traditional based curriculum are more knowledgeable in basic medicine such as disease process compared to their PBL curriculum medical graduates (Jones, McArdle and O'Neil, 2002; Dean et al., 2003). However, beyond the acquisition of the basic medical science knowledge, empirical evidence suggests that the supervisors thought that the graduates from the PBL curriculum are far well confident and indeed prepared for independent practice compared to the Non-PBL medical graduates (see for instance: Jones, McArdle\& O'Neil 2002; Dean et al., 2003; Marple, 2007; Alexander, 2007) etc. Their findings thus justify the inclusion of practice-based learning and improvement as an indicator of competence in medicine within the ACGME paradigm.

Practice-Based Learning and Improvement: Competence as a contextual concept especially from the point of view of the medical profession requires a practice-based training system. Such system should permit medical interns to spend most of their time providing patient care on a rather direct contact basis. In order to ensure the adequate preparedness of interns for independent practices, it is argued that that they should be more involved rather than detached from context specific medical situations (Epstein, 2007). This would not only enhance the exposure and experience of the interns, but also give their supervisors and other assessors an opportunity to evaluate their potentials for independent practice. Therefore, competence in medicine when assessed from the point of view of a habit connotes life-long learning which gives opportunities for selfappraisal and continuous improvement to the interns (Leach, 2002; Epstein, 2007). Hence, according to Marple (2007), competence of interns within the domain of problem based learning and improvement in the ACGME paradigm primarily evaluates the interns' ability to locate, appraise, and assimilate patients' medical presentation evidences. Subsequently, the interns should be able to analyze and apply their professional acumen based on practice and experience. This should always be with the intention of improving their competence via systematic methodology and statistical prowess to appraise clinical studies and related information on diagnostic and therapeutic effectiveness, and using available information technology to enhance their own knowledge base (Alexander, 2007).

Interpersonal Communication: Physicians, based on their human limitations acknowledge that they can only care as the power to cure resides in almighty Allah (God). This perhaps explains why the first indicator of competence in medicine is 'patient care'. Therefore, as it is the case at times, the efforts of the physician may only offer at best a palliative measure and yet this would have to be communicated to the patients or their guardians. According to Hurwitz and Vass (2002), patients are now perceptive than ever and want to have a say in their care. Patients derive satisfaction and perhaps this enhances quick healing (Janicik, et al., 2007), when physicians patiently listen to them and provide information and opportunities for participation in the patients' care. In cases like this, Janiciket al. (2007) noted that the physicians need to demonstrate communicative competence to deal with difficult patients like alcoholics, patients with terminal diseases, and patients from different cultures and religion as if it is the case in Malaysia, or even in worst scenarios announcing the demise of a patient to his relatives. Hurwitz and Vass (2002) found that patients seldom get such opportunities and interpersonal relationship. Even when patients prefer to have sufficient consultation, appointment at short notice, and detailed information about their ailment, physicians rarely have the time to do so. According to Marple (2007), the relative importance of effective communication and interpersonal skills as an indicator of competence in medicine perhaps explains why the PBL curriculum as being adopted in most medical schools emphasizes and views it (communication) as a cardinal competence. Janiciket al., (2007) argued further that medical supervisors opine that observing interns' performance beyond the intellectually rigorous process of clerking to include communication as they encounter patients with difficult communication challenges is most indicative of their preparedness for future challenges. The argument is that clerkship may only indicate proficiency in biomedical knowledge, which is content specific in difference to the context specific nature of modern healthcare realities. Furthermore, Watmough, Taylor, and Garden (2006) found that the supervisors' evaluation of the preparedness of interns for independent practice got very positive comments given the training in communication their supervisees had received. Therefore, Marple (2007) and Alexander (2007) argue that the interns are expected to demonstrate interpersonal and communication skills in a very scholarly manner (listening, non-verbal, explanatory, writing etc), in order to create a therapeutic and ethically sound relationship with patients and their families, and team members. 
Professionalism: The noble profession of medicine should ideally be taken up as a "calling" and only secondarily a fees-for-service or trade. Usually, a patient visits the hospital or a physician to seek prevention of; or medication to an ailment. When patients present with medical situations or complains relating to pain, injuries, or even imminent death, professionalism in medicine expects that the physician; while maintaining his composure also display professionalism especially as it relates to empathy for patients and other humanistic values (Shapiro, 2008). The demonstration of such professionalism as argued by Marple (2007) should ideally be through showing selflessness and compassion for the plight of the patients and as such, physicians should place their own personal interest below those of their patients and the society. Therefore, contrary to the social exchange theory that holds that the benefits must always outweigh the cost of an action to justify carrying out an activity, medicine as a profession entails the physician being altruistic in his conduct. Professionalism also entails accountability for own action as well as holding firmly to professional and medical ethos. Therefore, physicians have a duty of secrecy and confidentiality as it relates to the patients information at their disposal. Based on supervisors' perception of the interns on their demonstration of empathy to patients, Woolfeet al., (2007) found that houseman who had experience an illness themselves, in their friends or relatives are often than not more prepared to face the challenges of such ailment and also demonstrate high professionalism in doing so. This compares to Clay (1999) that new medical doctors opine that their personal illness experience influenced their empathy for patients and their relatives.

\section{Methodology}

Sample Size and Data Collection Method: At the point of conducting the study, the number of housemen attached to government hospitals was found to be 2,046. They were attached to government hospitals and the teaching hospitals of University Malaya, University Sains Malaysia and University Kebangsaan Malaysia. Due to the large geographical area to be covered, it was felt that the best data collection method was by selfadministered questionnaires. The list of hospitals and the number of questionnaires distributed is as in appendix 1. Given that the population under study was manageable, it was, therefore, decided that the questionnaires to be distributed to all the housemen throughout the country including Sabah and Sarawak. Since the geographical area covered is large, most of the questionnaires were sent by post. However, for some hospitals in the Klang Valley, such as Hospital Putrajaya and Hospital Kuala Lumpur, the questionnaires were delivered personally to the Hospital Director. From a total of 2,046 questionnaires sent out, a total of 1,278 responses were received. This gave a response rate of $62.46 \%$. Of these, 1,213 questionnaires were analyzed. Questionnaires, which were incomplete or considered outliers from the pattern of response, were excluded from the analyses. Thus, only data from properly completed questionnaires were used for the analyses, or $59.24 \%$. Outliers and questionnaires, which were not properly filled-in, made up only $5.16 \%$ of the response received. As per the outliers, with a Mahalanobis distance critical value of 13.82 for two independent variables lesser than the calculated value of 18.37, the impact should be minimal given the Cook's distance maximum value of 0.263 being lesser than 1 (Pallant, 2007). The data was subsequently subjected to normality test to determine whether or not it was normally distributed. The normality assumption tests include P-P plot and the scatter plot. The former indicate the non-normality of the data as some points do not lie on the straight diagonal line. Based on the Kolmogorov-Smirnov test, it was found that some items are not normally distributed at significance level of 0.05 . Nevertheless, this should not have any serious impact on the data given a large sample size (Pallant, 2007).

Respondents' Profile: The profile of the respondents is shown in Table 2 below. In terms of age, almost all of the respondents are under 30years, and about 60 percent among them are females. Also expected of their marital status, about 80 percent indicated they were single. Based on ethnic classification, more than half of the respondents are Malays, while one-fourth are Chinese. Interns of Indian descent represent about 16 percent of respondents. Moreover, more than half of the respondents are on government loan sponsorship. In terms of frequency, Table 2 above indicates that the leading medical schools in Malaysia have a representation of 55 per cent. Malaysian schools adopting the United Kingdom and Indian curricular have 13.1 per cent and 12.6 per cent respectively. Such UK curricula include those of Dublin, Ireland and Sheffield, Britain. The Indian curriculum is basically that of Manipal medical school. With almost equal representation with the UK and Indian curricula adopting Malaysian schools is the frequency for University of Malaya (UM) which has 12.4 per cent respondents. The distribution above which; though in aggregate represent about 66 
per cent of total respondents is nonetheless well distributed to avoid undue influence on the quality of analysis.

Development of the questionnaire: The questionnaire covered several key areas regarding the competence of a medical officer. Respondents were asked to indicate their response using a 5-point Likert scale. The dimensions measured by the questionnaire were derived from the research model provided by the Institute of Health Management on the pre-employment training of medical professionals. Table 2 below shows the reliability, as well as the sources of the research instruments used in the study.

Table 2: Demographic Distribution of Respondents

\begin{tabular}{lll}
\hline & Frequency (N) & Percentage (percentage) \\
\hline Age & 651 & 53.7 \\
$20-25$ & 534 & 44.1 \\
$26-30$ & 17 & 1.4 \\
$31-35$ & 10 & 0.8 \\
Others & & \\
Gender & 505 & 41.7 \\
Males & 707 & 58.3 \\
Females & & \\
Ethnic Background & & 54.9 \\
Malay & 665 & 25.5 \\
Chinese & 309 & 16.0 \\
Indian & 194 & 3.6 \\
Others & 44 & \\
Financing Mode & & 55.2 \\
Government & 669 & 29.8 \\
Self-Sponsored & 361 & 15.0 \\
Others & 182 & \\
Marital Status & & 83.2 \\
Single & 1,009 & 16.0 \\
Married & 194 & 10.0 \\
Others & 10 & \\
Curricula & & 55.0 \\
Malaysian Universities & 669 & 13.1 \\
Adapted UK* & 159 & 12.6 \\
Adapted India* & 153 & 4.5 \\
UK trained & 55 & 7.6 \\
Indonesia trained & 92 & 3.7 \\
Eastern Europe trained & 45 & 3.2 \\
Others & 39 &
\end{tabular}

* Adapted UK and Adapted India represents medical schools in Malaysia adopting UK and Indian Medical Schools' curricula.

Table 2: Scale development, source and reliability

\begin{tabular}{lll}
\hline Construct & Scale/Source & Reliability \\
\hline Preparedness for & Australian study (Hill et al. 1998), & $>0.80$ \\
Independent Practice & Paolo and Bonaminio (2003) & \\
\hline
\end{tabular}

Source: Authors' computation

Statistical Tool of Analysis: Structural Equation Modeling (SEM) is used to analyze the data for this study. SEM is a multivariate technique used to analyze hypothesized relationship specified on theoretical or empirical evidences, and transformed into path diagrams (Mueller and Hancock, 2008). As stated in Adewale (2010), SEM's graphical features not only allow for the analyses of latent factors just like in the regression analysis but also permit modification of theoretical models to capture varying interrelationships (Hair, et al, 
2006). Moreover, following Adewale (2010), SEM's focus on theoretical explanation rather than prediction, albeit it also captures the latter, suited well the objectives of this study. In all instances, a summated scale was used to arrive at the indicator variables. Following Adewale (2010), a partial aggregation method was used to arrive at the summated scales. ${ }^{2}$

\section{Results}

Measurement Model: A review of the Confirmatory Factor Analysis model based on the various criteria in SEM shows that there are no offending estimates ${ }^{3}$ and that the model fits well. The hypothesized measurement model was assessed using AMOS version 18.0 maximum likelihood factor analysis. The model was evaluated by four fit measures: a) the chi-square, b) the comparative fit index (CFI), the normed fit index (NFI), and the root mean square error of approximation (RMSEA) as per Meyers et al (2006) and Mueler and Hancock (2008). Results of all four fit indexes support the proposed model. The chi-square had a value of $16.958(6, \mathrm{~N}=1,213), p=0.009$, indicating a statistical significance. Model fit based on chi-square in SEM should not be statistically significant in order to indicate a good fit. However, given that the chi-square is highly susceptible to sample sizes, Mueller and Hancock (2008), Blunch (2008), suggested the normed chi-square (CMIN) should be used instead. ${ }^{4}$ With a CMIN value of 2.826, this is within the range of between ratios 3:1 as suggested in Hair et al (2006) and attests to the fit of the measurement model. Moreover, the baseline fit indices are also more than the 0.90 cut-off point specified in most SEM studies. In this case, the CFI $=0.998$, and NFI= 0.996, indicate good fit of the measurement model. With a RMSEA value of 0.039 (P Close= 0.773), this is also less than the cut-off point of 0.08 . This result is shown in Table 3 below.

Table 3: Tabular Presentation of Fit Indices Criteria Compared to Baseline Model Output

\begin{tabular}{ccc}
\hline Fit Indices & Recommended Threshold & Model Output \\
\hline CMINDF & $2 \geq$ CMINDF $\leq 5$ & 2.826 \\
P & P $\geq 0.05$ & 0.009 \\
CFI & CFI $\geq 0.90$ & 0.998 \\
NFI & TLI $\geq 0.90$ & 0.996 \\
RMSEA & RMSEA $\leq 0.08$ & 0.039 \\
\hline
\end{tabular}

Source: Authors' computation

Factorial Invariance: To test factorial invariance, a simultaneous analysis of the divides was carried out. For instance in terms of gender, analysis based on Male and Female grouping was carried out to determine the configural fit of the model ${ }^{5}$. In this case, all the factor loadings were unconstrained to be equal to each other across the groups (Male $=n 1=505$ ) and (Female $=n 2=708$ ). All the fit indices met the criteria for model fit. The chi-square test for differences was subsequently carried out to test the statistical significance of the moderating effect of gender on the measurement model. In this case, the five latent constructs were constrained while the indicators (questionnaire items) were allowed to load freely. It was revealed that the hypothesized model was not invariant between the two groups: male and female medical interns, $\chi^{2}(7, \mathrm{~N}=$ $1213)=26.112, p<0.01$. This result is shown in Table 4 below:

\footnotetext{
${ }^{2}$ Items related to a latent construct were subjected to an exploratory factor analysis. The average score of the items that have significant factor loadings was used to represent respective construct as an indicator in both the measurement and structural models.

${ }^{3} \mathrm{~A}$ direct path coefficient or regression coefficient with a value greater than 1.00. This is considered unacceptable in an SEM analysis.

${ }^{4} \mathrm{~A}$ normed chi-square is denoted by $\chi^{2} / \mathrm{df}$. That is, chi-square value divided by degrees of freedom. It is a goodness of fit (GOF) measure in SEM. According to Hair et al (2006:748), generally, $\chi^{2}: d f$ ratios on the order of 3:1 or less are associated with better fitting models except when sample size is greater than 750 . This measure also called the relative likelihood ratio was used to mitigate the susceptibility of chi square to spuriousness especially as sample size grows bigger (Firdaus, 2005). For this measure, a value of between 2 and 5 is considered acceptable (Sahari et al, 2004).

${ }^{5}$ Similar groupings were made for age (Below 40 years and Above 40 years); Occupation (Trading and Non-Trading); MSE type (Survivalists and Growth Oriented), Educational attainment (primary school and below, secondary school and above) etc.
} 
Table 4: Results of Multiple Group Modelling

\begin{tabular}{llccccc}
\hline Demographic Factor & Model & $\chi^{2}$ & Df & $\begin{array}{c}\text { Critical- } \\
\text { Value }\end{array}$ & $\boldsymbol{\Delta} \chi^{2}$ & Sig. \\
\hline Gender & Unconstrained & 26.112 & 12 & & & \\
Age & Constrained & 29.308 & 17 & 20.515 & 3.196 & N.S \\
Curricular & Unconstrained & 26.112 & 12 & & & \\
& Constrained & 29.236 & 17 & 20.515 & 3.124 & N.S \\
Financing Mode & Unconstrained & 26.112 & 12 & & & \\
& Constrained & 29.274 & 17 & 20.515 & 3.162 & N.S \\
& Constrained & 26.112 & 12 & & & \\
& Unconstrained & 29.365 & 17 & 20.515 & 3.253 & N.S \\
\hline
\end{tabular}

$P<0.001$ N.S. $=$ Non-Significant

As shown in Table 4 above, there is no statistically significant difference between the male and female groups' measurement models though they both have reasonable fit. In terms of age and financing mode and curricular, there was no sufficient evidence based on the data used in this study to conclude that there is a factorial invariance among the respondents.

\section{Conclusion}

The main objective of this study was to investigate what the core competence skills needed for post-medical school practice are as perceived by Malaysian medical interns. Based on the results of the measurement model via the structural equation modeling the indicators used in this study as proxy for various requisite skills for competent medical practice are all statistically significant. This is as per the responses of the interns in the study sample. Notwithstanding, the three most significant indicators are the interpersonal, basic, and patient management skills. A result that was rather unexpected is the statistical insignificance of the invariance analysis across demographic divides. It was also envisaged that the interns should at least show significant difference based on curricula. Nonetheless, the non-statistical significance of the invariance analysis across the divides should not be viewed in absolute terms. This is because the regression weight for each indicator skill in the model is not only significant in isolation but also changes as the demographic factors change. As such, a plausible conclusion may be that the medical profession requires a perfect blend of the attributes of an applied scientist and a medical humanist for a practitioner to demonstrate the requisite competence. Finally, the limitation in the findings of this study is well noted. As such, it is unlikely that there are other measures of competence in the medical profession. Howbeit exhaustive the skills identified in the study are, it is likely that there may be some invariance across demographic divides if same instrument is administered on a different sample.

\section{References}

Adewale, A. A. (2010). Localization of Microfinance as a Strategy to Alleviate Intergenerational Transmission of Poverty in Nigeria. An Unpublished Doctoral Thesis Submitted to the International Islamic university Malaysia.

Aggarwal, R., Grantcharov, T. P. \& Darzi, A. (2007). Framework for Systematic training and Assessment of Technical Skills. Journal of American College Surgeons, 1, 697-705.

Alexander, B. (2007). Assessing Resident Competency in Laboratory Medicine. Clinics in Laboratory Medicine, $27,269-281$.

Blunch, N. J. (2008). Introduction to Structural equation Modeling Using SPSS and AMOS. Singapore: Sage.

Clay, A. S. (1999). The Medical Student as a Patient. Ann. Intern Med., 131, 225-226.

Dean, S. J., Barratt, A. L., Hendry, G. D. \& Lyon, P. M. A. (2003). Preparedness for hospital practice among graduates of a problem-based, graduate entry medical program. Medical Education, 178, 163-178.

Epstein, R. M. (2007). Assessment in Medical Education. The New England journal of Medicine, 356(4), 387396. 
Hair, J. F. Jr., Anderson, R. E., Tatham, R. L. \& Black, W. C. (2006). Multivariate Data Analysis, (7th edn). Upper Saddle River, NJ: Prentice Hall.

Hurwitz, B. \& Vass, A. (2002).What is a good doctor, and how can you make one? BMJ, 325, 667-668.

Janicik, R., Kalet, A. L., Schwartz, M. D., Zabar, S. \& Lipkin, M. (2007). Using Bedside Rounds to teach Communication Skills in the Internal Medicine Clerkship. Medical Education Online (serial online), 12(1).

Jones, A., McArdle, P. \& O'Neill, P. (2002). Perceptions of how well graduates are prepared for the role of preregistration house officer: a comparison of outcomes from a traditional and an integrated PBL curriculum. Med Educ, 6, 16-25.

Leach, D. C. (2002). Competence is a Habit. Journal of American Medical Association, 287(2), 243-244.

Marple, B. F. (2007). Competency-Based Resident Education. Otolaryngologic Clinics of North America, 40, $1215-1225$.

McLaughlin, C. P. \& Kaluzny, A. D. (1999). Continuous Quality Improvement in Health Care. Gaithesburg, U.S.: Aspen Publishers.

Meyers, L. S., Gamst, G. \& Guarino, A. J. (2006). Applied Multivariate Research. London: Sage Publications Inc.

Mueler, R. O. \& Hancock, G. R. (2008). Best Practices in Structural Equation Modeling in J. W. Osborne (Ed). Best Practices in Quantitative Methods. London: Sage Publications.

Neeraj, K., Burkhalter, B. \& Cooper, M. (2001). Measuring the Competence of Healthcare Providers. Operations Research Issue Paper, 2(1).

Pallant, J. (2007). SPSS Survival Manual. Australia: Allen-Unwin.

Primadasa, I. G., Shehab, D., Al-Jarallah, K. F. \& Thabib, F. (2007). Confidence in Performing Core Clinical Skills: A Survey of Trainees Completing Housemenship in Kuwait. Bulletin of the Kuwait Institute for Medical Specialization, 6(68).

Shapiro, J. (2008). Walking a mile in their patients' shoes: empathy and othering in medical students' education. Philosophy, Ethics and Humanities in Medicine, 3(10), 1-11.

Sivalingam, N. (2004). Teaching and Learning of Professionalism in Medical Schools. Annals of Academic Medicine Singapore, 33, 706-710.

Wass, V. (2005). Ensuring medical students are fit for purpose. British Medical Journal, 331, 791-792.

Watmough, S., Taylor, D. \& Garden, A. (2006). Educational supervisors' evaluate the preparedness of graduates from a reformed UK curriculum to work as pre-registration house officers (PRHOs): a qualitative study. Medical Education, 40, 995-1001.

While, A. E. (1994). Competence versus performance: Which is more important? Journal of Advanced Nursing, 29, 525-31.

Woolf, K., Cave, J., McManus, C. \& Dacre, J. E. (2007). It gives you an understanding you can't get from any book.' The relationship between medical students' and doctors personal illness experiences and their performance: a qualitative and quantitative study. BMC Medical Education, 7(50), 1-9. 